\title{
Evolution of turbulence in an oscillatory flow in a smooth-walled channel: A viscous secondary instability mechanism
}

\author{
J. A. Cosgrove, ${ }^{1}$ J. M. Buick, ${ }^{2}$ and S. J. Tonge ${ }^{1}$ \\ ${ }^{1}$ School of Physics, The University of Edinburgh, Edinburgh EH9 3JZ, Scotland, United Kingdom \\ ${ }^{2}$ Department of Physics and Electronics, School of Biological, Biomedical and Molecular Sciences, University of New England, \\ New South Wales 2351, Australia \\ (Received 6 February 2003; published 5 August 2003)
}

\begin{abstract}
In this paper, a comparison is made of the evolution of turbulence in oscillatory channel flows with a zero-mean velocity in two and three dimensions, using the numerical technique of the lattice Boltzmann method. The results confirm a primary two-dimensional instability. Evidence is shown of a secondary, viscous three-dimensional instability mechanism acting in the oscillatory boundary layer, which is consistent with experimental observations.
\end{abstract}

DOI: 10.1103/PhysRevE.68.026302

\section{INTRODUCTION}

Oscillatory and pulsating flows occur in a variety of scales and applications ranging from naturally occurring examples, such as blood flow in arteries, air flow in the windpipe, or larger scale examples such as wave motion over sand beds in oceanographic studies to technological applications, such as the flow in engine cylinders and oscillatory chemical mixers. Unsteady flows can be either periodic (either pulsating or purely oscillatory) or aperiodic, for example, a flow undergoing sudden acceleration. Here, purely oscillatory flow with a zero-mean velocity in wall-bounded channels is examined. Besides being a relatively simple flow which has an exact analytical solution of the Navier-Stokes equation in the laminar regime, it also serves as a prototype problem from which conclusions can be inferred for more complicated nonperiodic flows.

The phenomenon of turbulence represents a continual challenge to theoreticians, numericists, and experimentalists and this is particularly the case when investigating purely oscillatory flows, where there is a difference between theoretical predictions [1-5] and experimental observations [6-11]. Evidently, numerical modeling can aid the understanding of this phenomenon and serve to bridge the disparity between the theory and the experiment. Despite this, there have been relatively few numerical studies performed relating to purely oscillatory flows and the examination of the evolution of turbulence, most notably Refs. $[2,12,13]$.

In this paper, we present the results of a direct numerical simulation of the evolution of three-dimensional turbulence in an oscillatory flow with a zero-mean velocity using the lattice Boltzmann method (LBM). The results are in agreement with experimental observations and provide evidence about the nature of the transition mechanism.

For the case of a fluid with a kinematic viscosity $\nu$ oscillating with an angular frequency $\omega$ in a channel with a halfwidth $a$, the relevant nondimensional parameters describing the main characteristics of the flow are the Womersley parameter $\alpha=a \sqrt{\omega / \nu}$ and the Reynolds number based on the Stokes layer thickness, $\operatorname{Re}_{\delta}=U_{0} \delta / \nu$. Here, $U_{0}$ is the maximum amplitude of the velocity and $\delta=\sqrt{2 \nu / \omega}$ is the Stokes layer thickness. The Stokes parameter $\Lambda$ is often used instead
PACS number(s): 47.27.Cn, 47.11. $+\mathrm{j}, 47.60 .+\mathrm{i}$

of $\alpha$ with $\Lambda=a / \delta=\alpha / \sqrt{2}$. Exact analytical solutions exist for laminar flows and these agree well with experimental results. Experimental studies of oscillatory flows with a zeromean velocity indicate that transition to turbulence occurs in the range $500<\operatorname{Re}_{\delta}<550$ at phases in the flow which correspond to the regions of maximum flow velocity. This transition phenomenon is clearly observable and consistent data have been published [6-11]. This is at odds with analytical stability analyses $[3,4]$. Theoretical analyses are hampered by a lack of derivations of the critical Reynolds number based on linear theories from which nonlinear theories can be developed. Local instabilities exist in the Stokes boundary layer and it is generally agreed, for problems where the boundary layer is small compared to the physical dimensions of the problem, that this is the cause of transition to turbulence. Therefore, for theoretical stability analyses, much of the work revolves around studying infinitesimal disturbances in the Stokes layer for which the equations of motion can be linearized. The problem is generally approached using Floquet or quasisteady theories $[1,3,4]$. In quasisteady theories, the spatially dependent profiles of the flow are examined at different instances of time and therefore the profiles have only a parametric dependence on time. This method differs from the time-dependent ones that use the Floquet theory to examine disturbances in the periodic steady state. However, these either do not predict the correct phase for the onset of turbulence or do not predict instability growth over a large range of $\operatorname{Re}_{\delta}$ and wave number [1].

The discord between the theory and the experiment has been explained by a secondary inviscid instability mechanism whereby the existence of finite-amplitude waves leads to vortical structures within the flow that are inviscidly unstable to three-dimensional perturbations. Pierrehumbert [14] argues that with regard to the transition to a developed turbulence spectrum, the short-wave behavior of the instability implies that the development of a full three-dimensional turbulence spectrum does not require that energy be handed down in a cascade from scale to scale until the dissipation range is reached, rather the large eddies provide a route whereby energy can be injected directly into the dissipation range. Therefore, the broadband nature of this instability provides a mechanism for the rapid generation and growth of small scales from a smooth velocity profile $[2,14,15]$. Akhavan, Kamm, and Shapiro [2] identified this secondary instability mechanism and suggested that it was inviscid, based 
on three-dimensional simulation results using a spectral method.

Previous (two-dimensional) theoretical studies have largely concentrated on examining the growth (or decay) rates of initial perturbations at critical phases within the cycle. Direct numerical simulations [12] of oscillatory flow between the walls with small imperfections have indicated that the imperfections play a fundamental role in triggering transition to turbulence and no mention is made in these studies of a secondary instability mechanism. In the current implementation, the wall is smooth with no imperfections. The inherent symmetry of the model is broken by perturbing the simulation by an excitation of all simulated wave numbers over the entire computational domain [16] and therefore in the context of the current study, imperfections in the wall would simply be interpreted as another method to perturb the inherent instabilities which exist in the flow. It is these instabilities which are of concern in this paper and in particular, evidence is shown that a secondary instability exists in the boundary layer.

\section{THE LATTICE BOLTZMANN MODEL}

The relative merits of the LBM are well established [17]; it lends itself naturally to parallel implementation, boundary conditions are easily applied, and it is easily interpreted in physical terms. The two-dimensional (three-dimensional) simulations were performed using the D2Q9 [18] (D3Q15 [18]) model where each grid site is connected to 8 (14) neighboring sites by the link vectors $\boldsymbol{e}_{i}$ on a square grid including diagonals (cubic grid with six vectors along the three axes of the cube and eight vectors along the diagonals); the final link direction is the null vector $\boldsymbol{e}_{0}$. The following description of the LBM is applicable to both models where the index $i$ is assumed to run from 0 to 8 ( 0 to 14$)$ for the D2Q9 (D3Q15) model.

The fluid is described in terms of the distribution functions $f_{i}(\boldsymbol{x}, t)$ from which the local fluid density and velocity can be determined as [19]

$$
\rho=\sum_{i} f_{i} \quad \text { and } \quad \rho u_{\alpha}=\sum_{i} f_{i} e_{i \alpha}+\frac{1}{2} F_{\alpha},
$$

where $\boldsymbol{F}$ is an external body force (per unit volume) and the Greek subscripts represent the vector components and the summation over repeated Greek indices is assumed. The distribution functions evolve according to the discrete Boltzmann equation $[19,20]$

$$
\begin{aligned}
f_{i}\left(\boldsymbol{x}+\boldsymbol{e}_{i}, t+1\right)-f_{i}(\boldsymbol{x}, t)= & \frac{-1}{\tau}\left[f_{i}(\boldsymbol{x}, t)-\bar{f}_{i}(\boldsymbol{x}, t)\right] \\
& +3 \frac{2 \tau-1}{2 \tau} w_{i} F_{\alpha} e_{i \alpha},
\end{aligned}
$$

where $\tau$ is the relaxation time and $w_{i}$ is a weight function with $w_{0}=\frac{16}{36}, w_{1}, \ldots, w_{4}=\frac{4}{36}$, and $w_{5}, \ldots, w_{8}=\frac{1}{36} \quad\left(w_{0}=\frac{16}{72}\right.$, $w_{1}, \ldots, w_{6}=\frac{8}{72}$, and $w_{7}, \ldots, w_{14}=\frac{1}{72}$ ) for the D2Q9 (D3Q15) model. The equilibrium distribution function $\bar{f}_{i}(\boldsymbol{x}, t)$ is determined from the local density and velocity as [17]

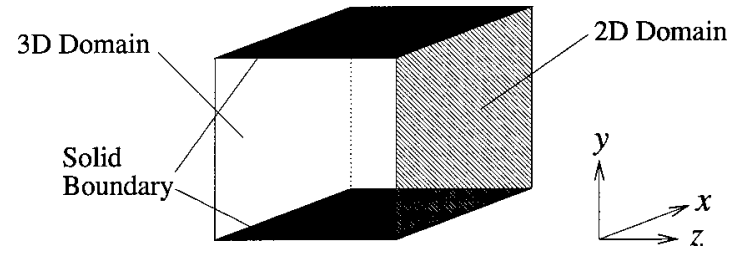

FIG. 1. Schematic diagram of the computational domain and coordinate system.

$$
\bar{f}_{i}(\boldsymbol{x}, t)=w_{i} \rho\left[1+3 \boldsymbol{e}_{i} \cdot \boldsymbol{u}+\frac{9}{2}\left(\boldsymbol{e}_{i} \cdot \boldsymbol{u}\right)^{2}-\frac{3}{2} u^{2}\right] .
$$

The distribution function can be expanded about the equilibrium value to obtain equations which represent the continuity and Navier-Stokes equation $[18,19]$ in the incompressible limit for a fluid with a kinematic viscosity that is determined by the relaxation time $\nu=(2 \tau-1) / 6$ and an equation of state $p=\rho c_{s}^{2}$, where $c_{s}=1 / \sqrt{3}$ is the speed of sound.

The computational domain is shown in Fig. 1, where the shaded $x-y$ plane is the two-dimensional region and the full volume is the three-dimensional domain. At the solid boundaries, a halfway bounce back boundary condition is applied such that any distribution function $f_{i}\left(\boldsymbol{x}, t^{-}\right)$, which is streaming from a "fluid" grid point to a "wall" grid point, is reflected as $f_{i^{\prime}}\left(\boldsymbol{x}, t^{+}\right)$, where $\boldsymbol{e}_{i^{\prime}}=-\boldsymbol{e}_{i}$ and $t^{-}$and $t^{+}$are the times just before and just after streaming, respectively. For planar surfaces aligned with one of the link vectors $\boldsymbol{e}_{i}$, this bounce back rule simulates a boundary which is halfway between the fluid and the wall sites and exhibits a secondorder accuracy $[16,21,22]$. When more complex boundaries are required in a simulation, the bounce back technique provides an efficient boundary treatment, however, an alternative approach is required to achieve the second-order accuracy, see, for example, Ref. [23] and references therein. Periodic boundary conditions are applied to all other boundaries. The flow is driven by an oscillatory pressure gradient which is implemented as an oscillatory body force $\boldsymbol{F}$ $=(P \sin (\omega t), 0,0)$ in Eqs. (1) and (2), where the values of $P$, the amplitude of the pressure gradient driving the flow, and $\omega$ are selected to give the desired values of $\operatorname{Re}_{\delta}$ and $\alpha$. This implementation of a body force can be shown to satisfy the continuity and Navier-Stokes equations up to second order $[16,19]$. To trigger the turbulence it is necessary to introduce a small random variation into the simulation in order to disturb the inherent symmetry. This was done by perturbing the forcing term to produce small irregularities in the flow with an energy of the order of $10^{-2}$, when normalized to the energy of the mean flow. The simulations were initialized using the analytic solution for the laminar case. This prevented transients in the flow and the same turbulent characteristics were observed in each half period with variations which were consistent with the turbulent nature of the flow rather than a systematic time-dependent variation originating from the initialization as found in Ref. [16]. A detailed description of the numerical technique applied in two dimensions can be found in Ref. [16].

For the current investigation, $\alpha=7$, giving a ratio of the half-channel width to the Stokes layer of $\Lambda=4.95$. This value of $\alpha$ was chosen as a compromise between keeping 

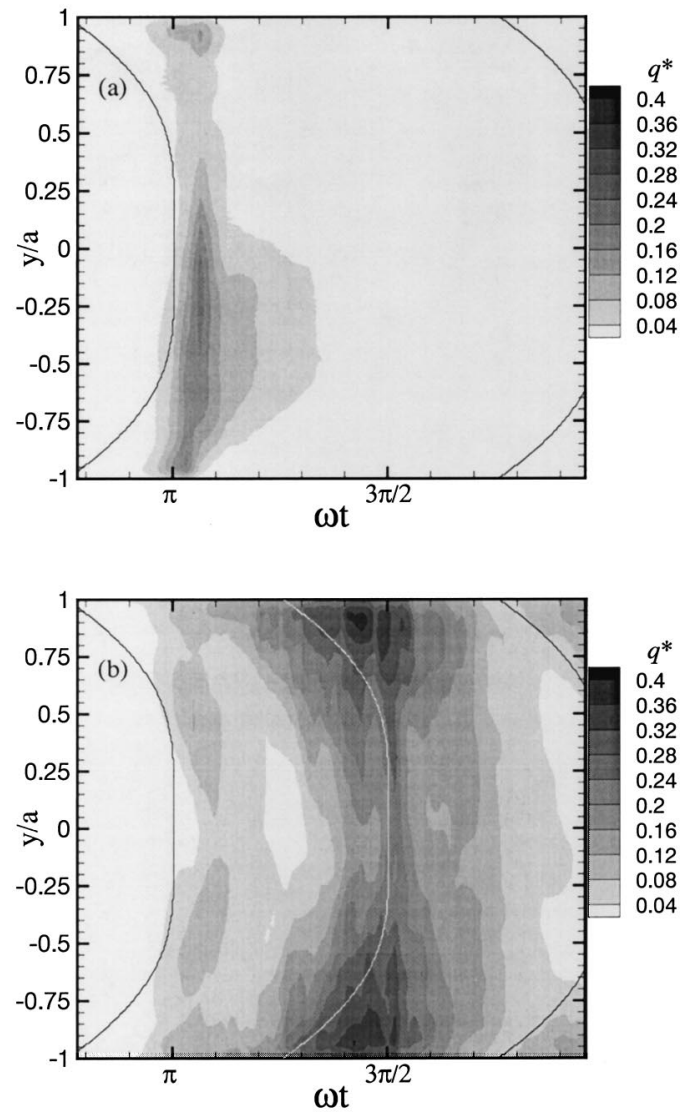

FIG. 2. Evolution of turbulent intensity across the width of a channel for (a) a two-dimensional and (b) a three-dimensional flow.

this ratio relatively large while obtaining a sufficient resolution in the boundary layer, in this case, 15 lattice nodes. We note that the resolution of the boundary layer is $\approx 2.5$ that of Akhavan's [2]. A single Reynolds number $\operatorname{Re}_{\delta}=850$ was chosen well above the critical range for transition to turbulence, $500<\operatorname{Re}_{\delta}<550$, and therefore the current investigation is not concerned with transition in the sense of finding critical Reynolds numbers, but with the generation and evolution of turbulent bursts in the flow at critical phases. Furthermore, critical scales associated with the vortices at the point of transition to turbulence at these phases are examined. First, a comparison is made between direct numerical simulations of a two-dimensional (2D) and a three-dimensional channel performed using the lattice Boltzmann model. In both cases, 148 grid points were used in the $y$ direction, between the channel walls, and 128 in the continuous directions.

\section{RESULTS AND DISCUSSION}

Figures 2(a) and 2(b) indicate the evolution of the normalized turbulent intensity $q^{*}=\sqrt{\left(\overline{u_{x}^{\prime 2}}+\overline{u_{y}^{\prime 2}}+\overline{u_{z}^{\prime 2}}\right) /}$ $\sqrt{\left({\overline{u_{x}}}^{2}+{\overline{u_{y}}}^{2}+{\overline{u_{z}}}^{2}\right)}$ over approximately half a period for both the $2 \mathrm{D}$ and $3 \mathrm{D}$ computational domains. The overbar represents averaging over all points with the same $y$ value and the prime superscripts indicate fluctuating values of velocity. Throughout the paper, a superscript * represents a dimensionless quantity with $x^{*}=x / a, y^{*}=y / a$, and $z^{*}=z / a$.
Note that in the two-dimensional case, $u_{z}^{\prime}=u_{z}=0$. Superimposed on the plots are the lines of zero velocity (black) and in Fig. 2(b) the maximum velocity magnitude, (white) which serve as the references of flow reversal and maximum velocity, respectively. Both these lines were calculated from the exact analytical solutions for laminar flow. A comparison of Figs. 2(a) and 2(b) indicates that the maximum turbulent intensities are generated at different phases for the twodimensional and three-dimensional simulations. In both cases, the initial disturbance field appears localized near the wall. In the two-dimensional case, the turbulent energy peaks are near the linear critical layer and neighboring inflexion points with the disturbances quickly migrating toward the center of the channel in agreement with quasisteady linear stability theories [13]. In the three-dimensional case, there is also an instability at $\omega t=\pi$, however, a significantly larger burst of turbulence is generated in the region of the maximum flow velocity at $\omega t=3 \pi / 2$ in agreement with previous experimental results. For the three-dimensional case, we note that the results are in good agreement with the experiment [6-11] in terms of the phase and relative channel locations at which rapid turbulence develops and we now proceed to examine the mechanism which generates this phenomenon.

Pierrehumbert [14] has investigated a short-wave instability of two-dimensional eddies in an inviscid fluid while Landman and Saffman [15] have extended this work for elliptical flow when the viscosity is nonzero. In this case, the nondimensional parameters of primary interest are the Ekman number $E_{\gamma}=4 \pi \nu k^{2} / \gamma$, where $E_{\gamma}$ is based on the vorticity $\gamma$ with $k$ the wave number and $\beta=2 \epsilon / \gamma=\left(\chi^{2}\right.$ $-1) /\left(\chi^{2}+1\right)$. The parameter $\beta$ is the ratio of strain $\epsilon$ to vorticity and $\chi$ is the aspect ratio of the elliptical streamlines. On performing a stability analysis of an elliptical flow which is an exact solution of the Navier-Stokes equations [15], a stability boundary in the Ekman number versus streamline eccentricity plane can be found. Considering a viscous instability with a length scale $l$, two bounds can be defined. A lower bound $l_{0}$ associated with the wave number, $l_{0}$ $=2 \pi / k_{0}$, defines the minimum length scale a disturbance requires and in the context of the current investigation, the macroscopic length scale of interest is $\delta$ which represents the upper limit of the length scale so that $l_{0} \leqslant l \leqslant \delta$. It is convenient to normalize the length scale to the Stokes boundary layer thickness $l^{*}=l / \delta$. Figure 3 displays the $z$ component of the dimensionless vorticity, $\gamma^{*}=\gamma a / U_{0}$, as a function of $\left(x^{*}, y^{*}, z^{*}=Z^{*} / 2\right)$ over half the channel width $\left(0 \leqslant y^{*} \leqslant 1\right)$ at selected phases of the oscillation period. The $x$ component of $\gamma^{*}$ is shown in Fig. 4 as a function of $\left(x^{*}\right.$ $\left.=X^{*} / 2, y^{*}, z^{*}\right)$ at the same phases as Fig. 3. Here, $X^{*}$ and $Z^{*}$ are the dimensionless grid lengths in the continuous $x$ and $z$ directions, respectively. A comparison of Figs. 3 and 4 determines the extent to which the vortices are two dimensional or three dimensional.

At $\omega t=1.06 \pi$ [Fig. 3(a)], $\gamma_{x}^{*}$ is significantly smaller than $\gamma_{z}^{*}$, indicating that the circulations are predominantly two dimensional. Here, vortices in the boundary layer $\left(y^{*}\right.$ $>0.8$ ) are sheared and streaks of vorticity can be observed. Treating these as ellipses and with an ellipse aspect ratio $\chi$ 


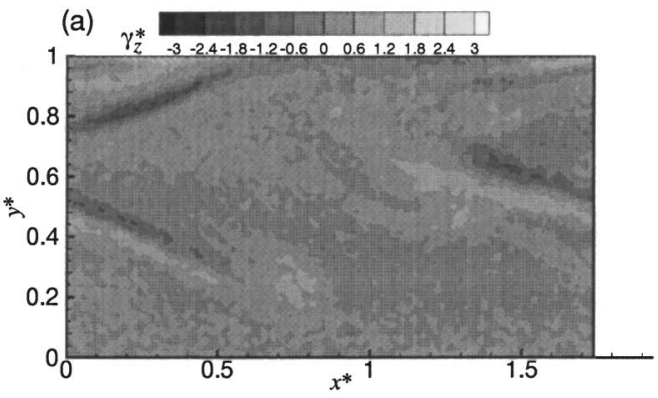

(b)

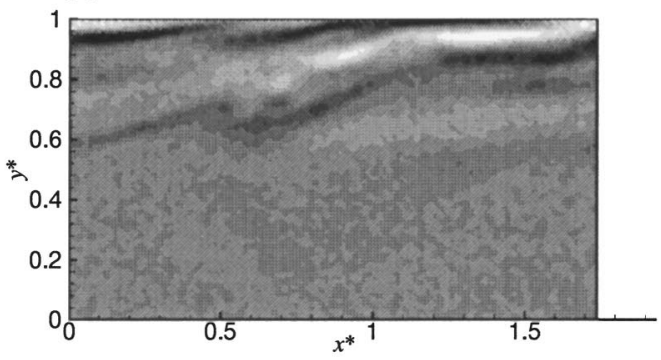

(c)

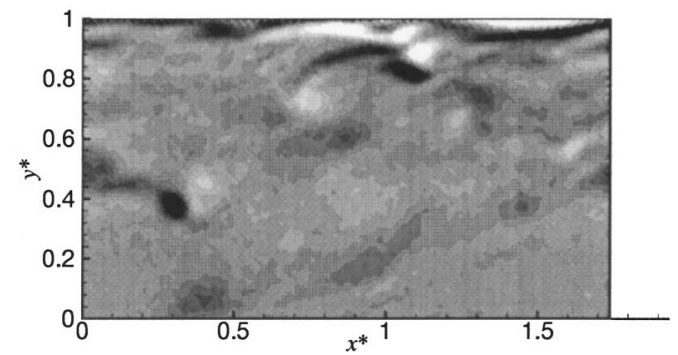

(d)

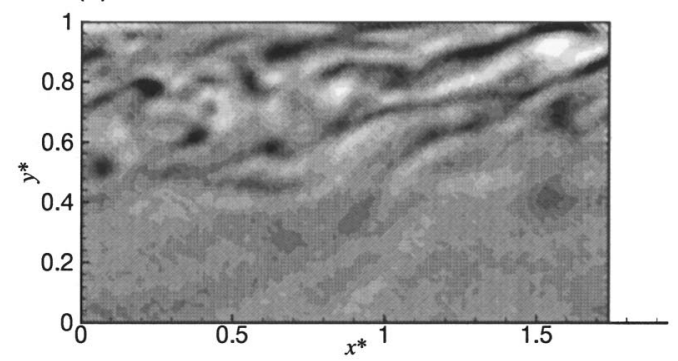

(e)

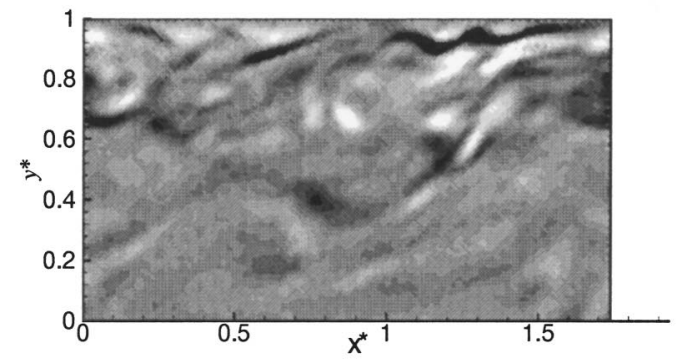

FIG. 3. Normalized vorticity calculated from the threedimensional simulations at $z^{*}=Z^{*} / 2$ for (a) $\omega t=1.06 \pi$, (b) $\omega t$ $=1.22 \pi$, (c) $\omega t=1.33 \pi$, (d) $\omega t=1.38 \pi$, and (e) $\omega t=1.45 \pi$.

$\approx 3$, a length scale $l_{0}^{*} \sim 2$ is obtained. Therefore, the secondary instability mechanism appears unlikely to be responsible for the transient growth and spread of the turbulence at this phase but rather is caused by the primary instability as described in, for example, Refs. $[2,16]$. However, this primary

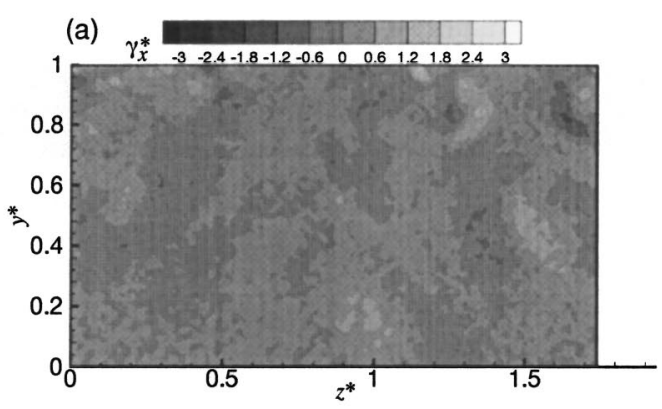

(b)

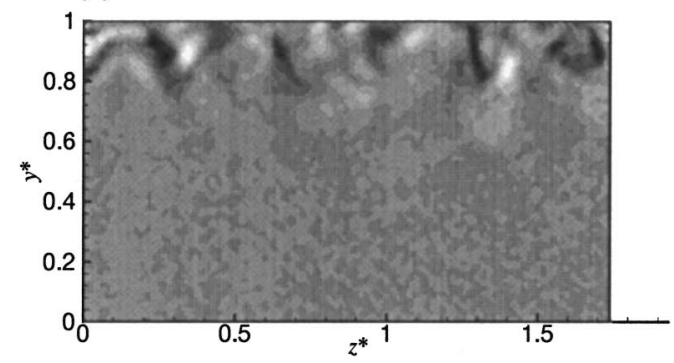

(c)

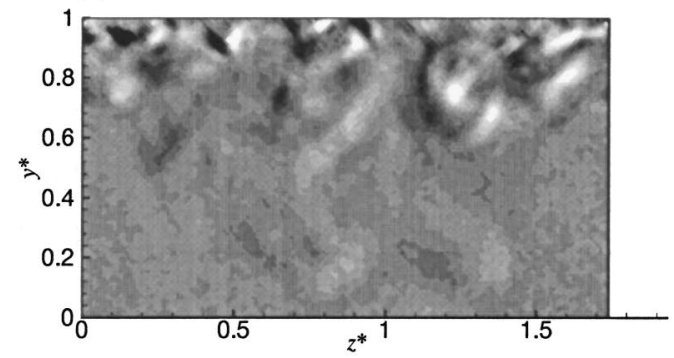

(d)

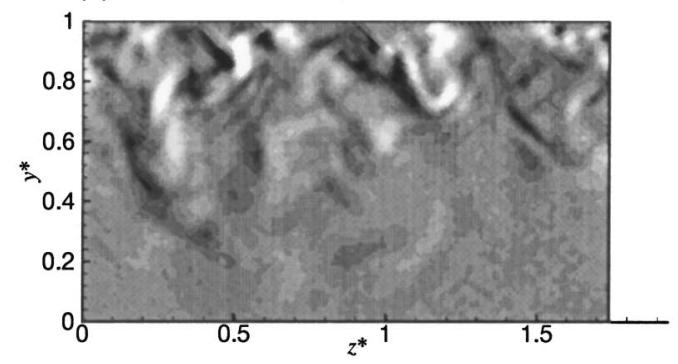

(e)

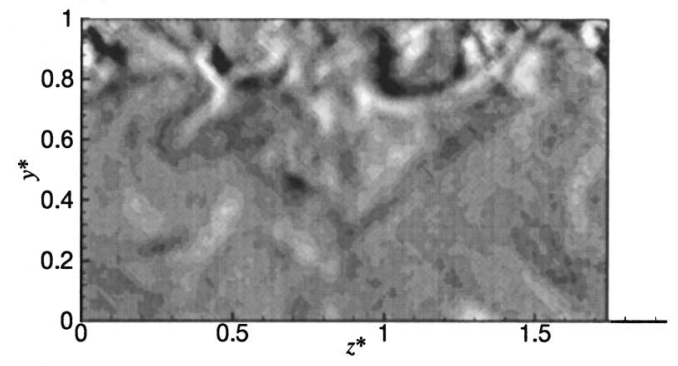

FIG. 4. Normalized vorticity calculated from the threedimensional simulations at $x^{*}=X^{*} / 2$ for (a) $\omega t=1.06 \pi$, (b) $\omega t$ $=1.22 \pi$, (c) $\omega t=1.33 \pi$, (d) $\omega t=1.38 \pi$, and (e) $\omega t=1.45 \pi$.

instability is fundamental to the development of vortices in the boundary layer and these persist and increase in vorticity as the flow accelerates as shown in Fig. 3(b) $(\omega t=1.22 \pi)$. Here, the magnitude of $\gamma_{x}^{*}$ is comparable although slightly smaller than $\gamma_{z}^{*}$ in the boundary layer, indicating that the 
three-dimensional motion is developing in the boundary layer. This three-dimensional motion is in part due to the vortices in the boundary layer tilting about the $y$ and $z$ axes and in part due to a rotation (predominantly in the $y$-z plane) along the major axes of the vortices. The two-dimensional circulations outwith the boundary layer persist and appear to pull the turbulence within the Stokes layer into the main flow further tilting the vortices in the boundary layer region. At this phase $(\omega t=1.22 \pi)$, a length scale of $l_{0}^{*} \sim 1$ is obtained based on an aspect ratio of $\chi \approx 3$. We note that $l_{0}^{*}$ is the same order of magnitude as $\delta$ and indeed there is no evidence of rapid three-dimensional transition at this phase [Fig. 2(b)]. The increase in turbulent intensity appears to be a result of the tilting and rotational motion of the originally twodimensional vortices in the boundary layer.

The continual stretching and tilting break up the elongated vortices forming a range of elliptical length scales which can be observed at $\omega t=1.33 \pi$ [Fig. 3(c)]. Here, $l_{0}^{*}$ reaches minimum values $\approx 0.5$ and is now approaching the correct order of magnitude enabling short-wave instabilities to exist. We note that this is the point just prior to the onset of the rapid transition to fully developed three-dimensional turbulence. As the flow further increases in velocity, $\omega t=1.38 \pi$ [Fig. $3(\mathrm{~d})], l_{0}^{*}$ reaches minimum values of $\approx 0.4$. From Fig. $2(\mathrm{~b}$ ), we note that this phase represents the region in the flow of the rapid increase and spreading of turbulent intensity, and indeed three-dimensional, approximately spherical, vortices with radii $r \leqq 0.25 \delta$ exist, two-dimensional slices of which can be seen in Figs. 3(d) and 4(d). This phase in the flow corresponds to maximum velocity in the bulk flow within the boundary layer. As the flow begins to decelerate, Figs. 3(e) and $4(\mathrm{e})$, at $\omega t=1.45 \pi$, the turbulence is fully three dimensional and extends toward the center of the channel.

The phase at which a rapid transition to turbulence is observed in the current investigation is consistent with previous numerical investigations $[2,13]$ and previous experimental results [6-11]. Costamagna, Vittori, and Blondeaux [13] observe similar behavior for the values of $\operatorname{Re}_{\delta}$ comparable to that used in the present study in terms of the initial generation of vortical streaks in the boundary layer [Fig. $3(b)$, which rotate in a similar manner as previously discussed and which move into the bulk flow [13].

Thus the initial generation of fluctuations is caused by the primary instability at flow reversal. These vortices persist in the boundary layer, shearing, stretching and tilting, and inevitably breaking up. In the region of maximum flow velocity, this process yields vortices of a size enabling short-wave instabilities to exist and it is at this region where a rapid transition to fully developed three-dimensional turbulence occurs. This rapid transition occurs in the boundary layer and propagates into the bulk flow.

\section{CONCLUSION AND FUTURE WORK}

In conclusion, the LBM simulations of two-dimensional flow were consistent with two-dimensional linear stability analyses, while the three-dimensional simulations were in good agreement with the experimental observations. Evidence has been presented to support the existence of a viscous secondary instability mechanism. Further analysis of this phenomenon over a wide range of $\alpha$ and $\operatorname{Re}_{\delta}$ is required to clarify the precise role of this instability mechanism in generating explosive turbulent growth in oscillatory flows.

\section{ACKNOWLEDGMENT}

This work was partially supported by EPSRC (UK) under Grant No. GR/N16778 and this assistance is gratefully acknowledged.
[1] C. V. Kerczek and S. H. Davis, J. Fluid Mech. 62, 753 (1974).

[2] R. Akhavan, R. D. Kamm, and A. H. Shapiro, J. Fluid Mech. 225, 423 (1991).

[3] P. A. Monkewitz and A. Bunster, in Stability of Time Dependent and Spatially Varying Flows, edited by D. L. Dwoyer and M. Y. Hussaini (Springer-Verlag, New York, 1987), pp. 244260.

[4] S. J. Cowley, in Stability of Time Dependent and Spatially Varying Flows (Ref. [3]), pp. 261-275.

[5] P. J. Blennerhassett and A. P. Bassom, J. Fluid Mech. 464, 393 (2002).

[6] P. Merkli and H. Thomann, J. Fluid Mech. 68, 567 (1975).

[7] M. Hino, M. Sawamoto, and S. Takasu, J. Fluid Mech. 75, 193 (1976).

[8] M. Ohmi, M. Iguchi, K. Kakehachi, and T. Masuda, Bull. JSME 25, 365 (1982).

[9] M. Hino, M. Kashiwayanagi, A. Nakayama, and T. Hara, J. Fluid Mech. 131, 363 (1983).

[10] M. H. A. Sinada, Ph.D. thesis, University of Edinburgh, 1984.

[11] R. Akhavan, R. D. Kamm, and A. H. Shapiro, J. Fluid Mech.
225, 395 (1991).

[12] G. Vittori and R. Verzicco, J. Fluid Mech. 371, 207 (1998).

[13] P. Costamagna, G. Vittori, and P. Blondeaux, J. Fluid Mech. 474, 1 (2003).

[14] R. T. Pierrehumbert, Phys. Rev. Lett. 57, 2157 (1986).

[15] M. J. Landman and P. G. Saffman, Phys. Fluids 30, 2339 (1987).

[16] J. A. Cosgrove, J. M. Buick, S. J. Tonge, C. G. Munro, C. A. Greated, and D. M. Campbell, J. Phys. A 36, 2609 (2003).

[17] S. Chen and G. D. Doolen, Annu. Rev. Fluid Mech. 30, 329 (1998).

[18] Y. H. Qian, D. d'Humieres, and P. Lallemand, Europhys. Lett. 17, 479 (1992).

[19] J. M. Buick and C. A. Greated, Phys. Rev. E 61, 5307 (2000).

[20] S. Chen, H. Chen, D. Martinez, and W. Matthaeus, Phys. Rev. Lett. 67, 3776 (1991).

[21] S. Chen and D. Martinez, Phys. Fluids 8, 2527 (1996).

[22] X. He, Q. Zou, L.-S. Luo, and M. Dembo, J. Stat. Phys. 87, 115 (1997).

[23] R. Verberg and A. J. C. Ladd, Phys. Rev. E 65, 016701 (2001). 